\title{
Supergiant Fast X-ray Transients with Swift: spectroscopic and temporal properties
}

\author{
P. Romano*, V. Mangano*, L. Ducci ${ }^{\dagger}$, P. Esposito**, R. Farinelli ${ }^{\ddagger}$, \\ C. Ceccobello ${ }^{\ddagger}$, S. Vercellone*, D.N. Burrows ${ }^{\S}$, J.A. Kennea ${ }^{\S}$, \\ H.A. Krimm, ${ }^{\mathbb{T}}$ and N. Gehrels ${ }^{\mathbb{I}}$ \\ *Istituto di Astrofisica Spaziale e Fisica Cosmica, Via U. La Malfa 153, I-90146 Palermo, Italy \\ ${ }^{\dagger}$ Institut für Astronomie und Astrophysik, Universität Tübingen, Sand 1, D-72076 Tübingen, \\ Germany \\ ${ }^{* *}$ Istituto di Astrofisica Spaziale e Fisica Cosmica, Via E. Bassini 15, I-20133 Milano, Italy \\ ${ }^{\ddagger}$ Dipartimento di Fisica, Università di Ferrara, via Saragat 1, 44122, Ferrara, Italy \\ ${ }^{\S}$ Department of Astronomy \& Astrophysics, Pennsylvania State University, University Park, PA \\ 16802, USA \\ "IIASA/Goddard Space Flight Center, Greenbelt, MD 20771, USA
}

\begin{abstract}
Supergiant fast X-ray transients (SFXTs) are a class of high-mass X-ray binaries with possible counterparts in the high energy gamma rays. The Swift SFXT Project ${ }^{1}$ has conducted a systematic investigation of the properties of SFTXs on timescales ranging from minutes to years and in several intensity states (from bright flares, to intermediate intensity states, and down to almost quiescence). We also performed broad-band spectroscopy of outbursts, and intensity-selected spectroscopy outside of outbursts. We demonstrated that while the brightest phase of the outburst only lasts a few hours, further activity is observed at lower fluxes for a remarkably longer time, up to weeks. Furthermore, we assessed the fraction of the time these sources spend in each phase, and their duty cycle of inactivity. We present the most recent results from our investigation. The spectroscopic and, most importantly, timing properties of SFXTs we have uncovered with Swift will serve as a guide in search for the high energy emission from these enigmatic objects.
\end{abstract}

Keywords: X-rays: binaries - X-rays: individual: IGR J16479-4514, XTE J1739-302, IGR J17544-2619, AX J1841.0-0536.

PACS: $97.80 . J p-98.70 . Q y$

\section{SUPERGIANT FAST X-RAY TRANSIENTS}

Supergiant fast X-ray transients (SFXT) are detected as fast hard X-ray transients whose hour-long outbursts reach $10^{36}-10^{37} \mathrm{erg} \mathrm{s}^{-1}$ and whose spectra resemble those of accreting neutron stars (NS). Indeed, some SFXT are shown to have pulsations ranging from a few seconds to $\sim 1000 \mathrm{~s}$. This flaring gives SFXTs quite a large dynamical range, which ranges between 3 and 5 orders of magnitude in flux. Optical identification of the counterparts has led to classify SFXTs as a peculiar class of high mass X-ray binaries (HMXB), associated to a blue (OB) supergiant companion. We generally distinguish between confirmed and candidate SFXTs based on the availability of an optical classification of the companion, and we now have 10 confirmed and as many candidate SFXTs. The details

${ }^{1}$ SFXT Project Page: http: //www.ifc.inaf.it/sfxt/ 


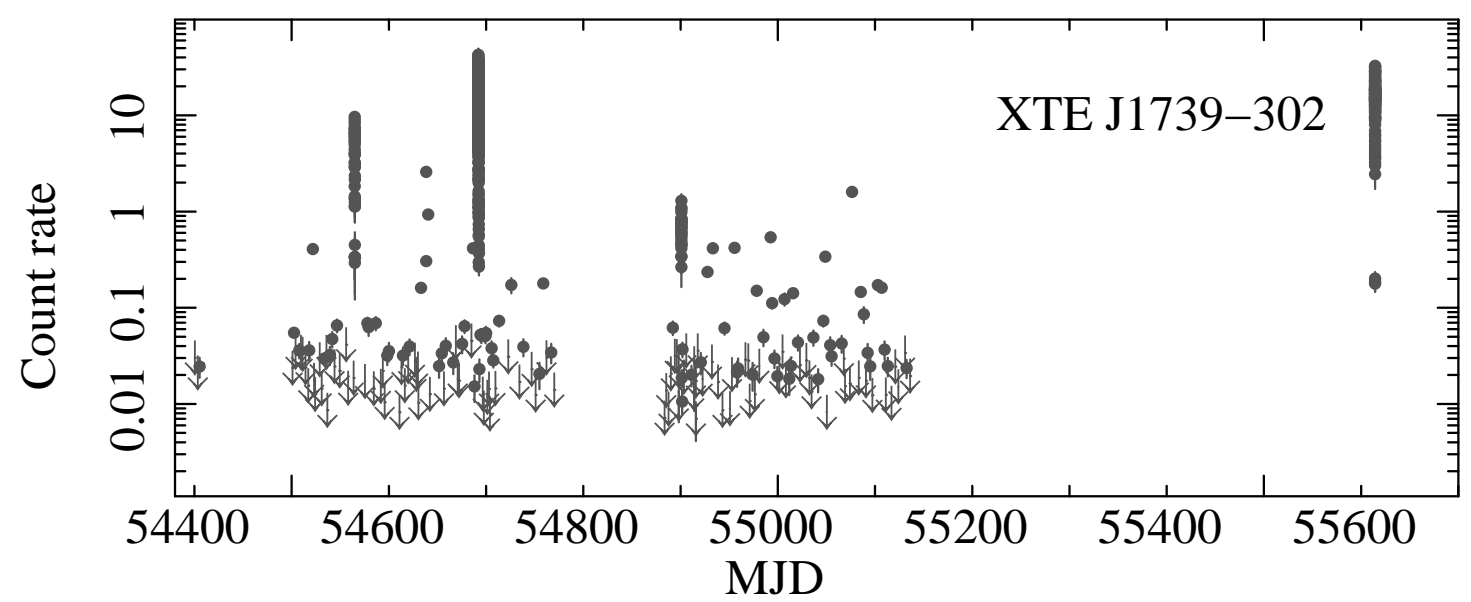

FIGURE 1. Swift/XRT (0.2-10 keV) long-term light curves of XTE J1739-302 and subsequent outbursts. The downward-pointing arrows are $3 \sigma$ upper limits. Adapted from [8].

of the physical origin of the outbursts are still unclear, but the ingredients that need to be taken into account include the properties of the wind from the supergiant companion $[1,2]$ and the presence of a centrifugal or magnetic barrier $[3,4]$.

\section{THE SFXT PROJECT: RECENT RESULTS}

Since 2007 the SFXT Project has performed an investigation of the properties of SFXTs with Swift, whose fast-slewing capability and broad-band energy coverage, combined with its flexible observing scheduling, make it an ideal facility to study both the bright outburst and the out-of-outburst behavior. Our strategy pairs monitoring programs with outburst follow-ups (see, e.g. [5]) and has allowed us to catch several outbursts from most confirmed and several candidate SFXTs (see, e.g. [6] and references therein), to monitor them during their evolution, and to study the long term properties of this class of objects with a highly sensitive soft X-ray telescope.

Our long-term monitoring of 4 SFXTs (IGR J16479-4514, XTE J1739-302, IGR J17544-2619, AX J1841.0-0536) has allowed us to determine that they spend $3-5 \%$ of the time in bright outbursts; that their most probable flux level is $\mathrm{F}(2-$ $10 \mathrm{keV}) \sim 1-3 \times 10^{-11} \mathrm{erg} \mathrm{cm}^{-2} \mathrm{~s}^{-1}$ (unabsorbed, $L \sim 10^{33}-10^{34} \mathrm{erg} \mathrm{s}^{-1}$ ) that their duty cycle of inactivity [7] is in the range $19-55 \%$, so that these sources accrete matter for most of the time [7]. Fig. 1 and 2 illustrate our long-term monitoring for the two SXFT prototypes, XTE J1739-302 and IGR J17544-2619 (up to MJD 55150) and how we keep observing bright outbursts also after the end of the campaign.

Further monitoring programs aimed at SFXTs with known orbital period. The first campaign was on IGR J18483-0311 [9] $\left(P_{\text {orb }} \sim 18.5 \mathrm{~d}\right)$ which we followed for a whole orbital period, thus allowing us to constrain the different mechanisms proposed to explain the SFXT nature. In particular, we applied the clumpy wind model for blue supergiants [10] to the observed X-ray light curve. By assuming an eccentricity of $e=0.4$, we could explain the X-ray emission in terms of the accretion from a spherically 


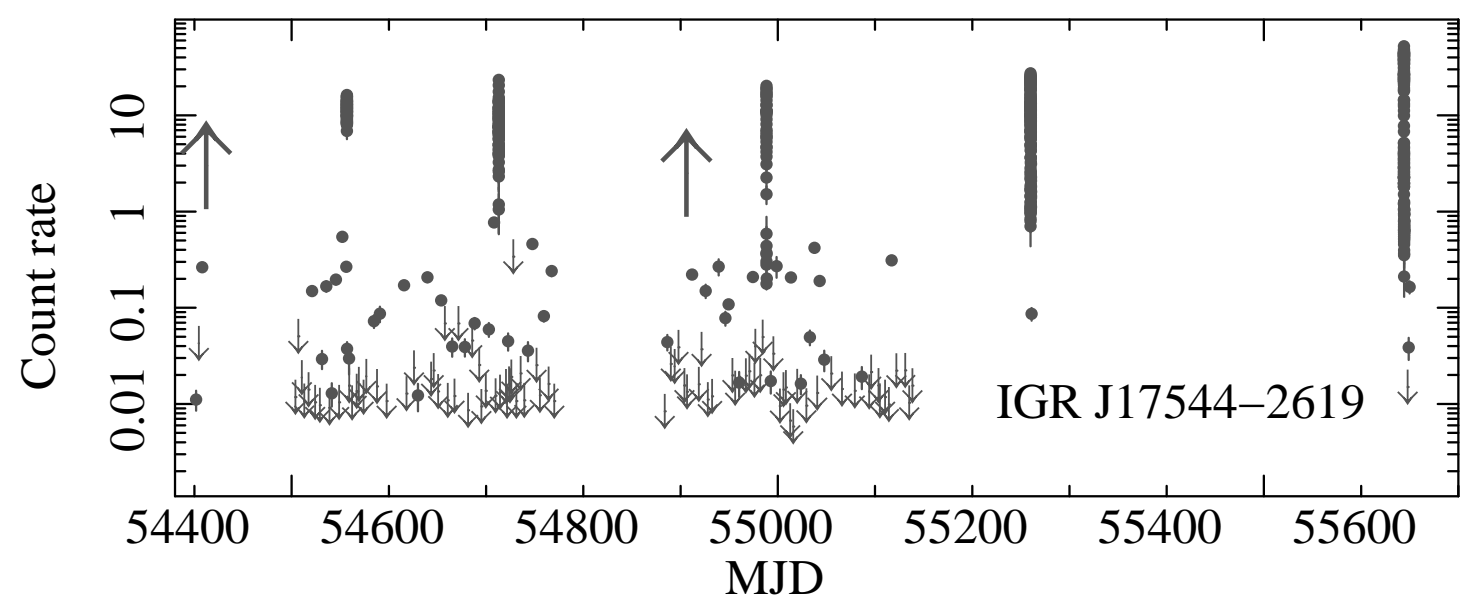

FIGURE 2. Swift/XRT (0.2-10 keV) long-term light curves of IGR J17544-2619 and subsequent outbursts. The downward-pointing arrows are $3 \sigma$ upper limits. The upward-pointing arrows mark flares that triggered the BAT Transient Monitor on MJD 54414 and 54906. Adapted from [8].

symmetric clumpy wind, composed of clumps with different masses, ranging from $10^{18}$ to $\times 10^{21} \mathrm{~g}$. We also monitored IGR J16418-4532 $\left(P_{\text {orb }} \sim 3.7 \mathrm{~d}\right)$ for three orbital cycles [11] and could account for the observed emission with accretion from a spherically symmetric clumpy wind (see, also [12]).

Among the most recent outbursts are those of XTE J1739-302 and IGR J17544-2619 that triggered the Swift/BAT on 2011 February 22 and March 24, respectively (Fig. 1, last peaks at MJD 55600, [8]) and those of IGR J08408-4503 [13].

In [8], we find that the spectra of both sources can be well fitted either with a twoblackbody model, or with a single unsaturated Comptonization model. In the latter case, the model can be either a classical static Comptonization model, such as COMPTT, or the recently developed COMPMAG model, which includes thermal and bulk Comptonization for cylindrical accretion onto a magnetized neutron star.

We refer to [14] for a detailed description of the algorithm. Here we briefly remind the reader that COMPMAG is based on the solution of the radiative transfer equation for the case of cylindrical accretion onto the polar cap of a magnetized NS. The velocity field of the accreting matter can be increasing towards the NS surface, or it may be described by an approximate decelerating profile. In the former case, the free parameters are the terminal velocity at the NS surface, $\beta_{0}$, and the index of the law $\beta(z) \propto z^{-\eta}$, while in the second case the law is given by $\beta(\tau) \propto-\tau$. The other free parameters of the model are the temperature of the black-body seed photons, $k T_{\mathrm{bb}}$, the electron temperature and vertical optical depth of the Comptonization plasma $k T_{\mathrm{e}}$ and $\tau$, respectively, and the radius of the accretion column $r_{0}$, in units of the NS Schwarzschild radius. The different combinations of these parameters determine the steepness of the spectrum at high energies and the rollover energy position. Fig. 3 shows the unfolded $E F(E)$ models and spectra for the COMPMAG model. 

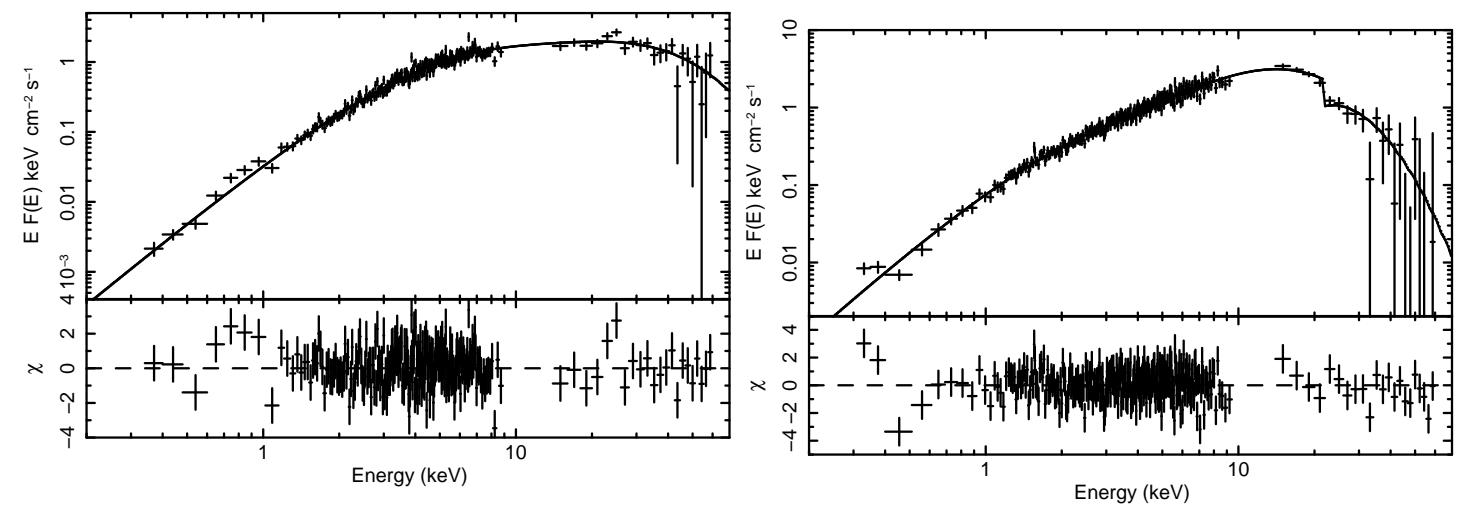

FIGURE 3. Left panel: Absorption-corrected unfolded $\mathrm{EF}(\mathrm{E})$ models and spectra, and residuals between the data and the COMPMAG model in units of $\sigma$ for XTE J1739-302. Right panel: Absorptioncorrected unfolded $\mathrm{EF}(\mathrm{E})$ models and spectra, and residuals between the data and the COMPMAG model in units of $\sigma$ for IGR J17544-2619. Adapted from [8].

\section{ACKNOWLEDGMENTS}

We acknowledge financial contribution from the contracts ASI-INAF I/009/10/0 and $\mathrm{I} / 004 / 11 / 0$.

\section{REFERENCES}

1. J. J. M. in't Zand, A\&A 441, L1-L4 (2005), astro-ph / 0508240.

2. L. Sidoli, P. Romano, S. Mereghetti, A. Paizis, S. Vercellone, V. Mangano, and D. Götz, A\&A 476, 1307-1315 (2007), 0710.1175.

3. S. A. Grebenev, and R. A. Sunyaev, Astronomy Letters 33, 149-158 (2007).

4. E. Bozzo, M. Falanga, and L. Stella, ApJ 683, 1031-1044 (2008).

5. P. Romano, S. Vercellone, V. La Parola, et al. "The Swift Supergiant Fast X-ray Transients Project: recent results," in Proceedings of the 25th Texas Symposium on Relativistic Astrophysics, Online at http://pos.sissa.it/cgi-bin/reader/conf.cgi?confid=123,2010, p. 117, 1103.6036.

6. P. Romano, V. Mangano, G. Cusumano, et al. MNRAS 412, L30-L34 (2011), 1012.0028.

7. P. Romano, V. La Parola, S. Vercellone, et al. MNRAS 410, 1825-1836 (2011), 1009.1146.

8. R. Farinelli, P. Romano, V. Mangano, et al. MNRAS 424, 2854-2863 (2012), 1205 . 7059.

9. P. Romano, L. Sidoli, L. Ducci, et al. MNRAS 401, 1564-1569 (2010), 0909.5109.

10. L. Ducci, L. Sidoli, S. Mereghetti, A. Paizis, and P. Romano, MNRAS 398, 2152-2165 (2009), 0906.3185.

11. P. Romano, V. Mangano, L. Ducci, et al. MNRAS 419, 2695-2702 (2012), 1109.6165.

12. P. Esposito, P. Romano, V. Mangano, L. Ducci, and S. Vercellone, "Swift monitoring of IGR J164184532," in These Proceedings, 2012, 1209.5872.

13. V. Mangano, P. Romano, C. Ceccobello, and R. Farinelli, "IGR J08408-4503 in outburst observed by Swift," in These Proceedings, 2012.

14. R. Farinelli, C. Ceccobello, P. Romano, and L. Titarchuk, A\&A 538, A67 (2012), 1111 . 6851. 